CERN-TH/99-46 BICOCCA-FT-99-05 IMPERIAL/TP/98-99/42 NYU-TH/98/2/03

hep-th/9903026

\title{
Confinement and Condensates Without Fine Tuning in Supergravity Duals of Gauge Theories
}

\author{
L. Girardello ${ }^{a, b}$, M. Petrini ${ }^{c}$, M. Porrati ${ }^{a, d}$ and A. Zaffaroni ${ }^{a}$ \\ (a)Theory Division CERN, Ch 1211 Geneva 23, Switzerland \\ (b)Dipartimento di Fisica, Università di Milano-Bicocca and INFN, Sezione di Milano, Italy] \\ (c) Theoretical Physics Group, Blackett Laboratory, Imperial College, London SW7 2BZ, U.K. \\ (d)Department of Physics, NYU, 4 Washington Pl., New York, NY 10003, USA
}

\begin{abstract}
We discuss a solution of the equations of motion of five-dimensional gauged type IIB supergravity that describes confining $S U(N)$ gauge theories at large $N$ and large 't Hooft parameter. We prove confinement by computing the Wilson loop, and we show that our solution is generic, independent of most of the details of the theory. In particular, the Einstein-frame metric near its singularity, and the condensates of scalar, composite operators are universal. Also universal is the discreteness of the glueball mass spectrum and the existence of a mass gap. The metric is also identical to a generically confining solution recently found in type $0 \mathrm{~B}$ theory.
\end{abstract}

CERN-TH/99-46

February 1999

\footnotetext{
${ }^{1}$ e-mail: girardello@milano.infn.it, m.petrini@ic.ac.uk, massimo.porrati@nyu.edu, alberto.zaffaroni@cern.ch

${ }^{2}$ Permanent Address

${ }^{3}$ Permanent Address
} 


\section{Introduction}

The duality between supergravity theories and strongly coupled field theories at large $N$ and large 't Hooft parameter [1] applies both to conformal [2, 3, 4] and non-conformal field theories [5, 6.

In order to be able to trust the supergravity approximation, one finds, quite generally, that the 't Hooft coupling, $x=N g_{Y M}^{2}$, must be large. In non-conformally invariant theories, where $x$ runs, this is to be understood as $x$ at the ultraviolet cutoff. This means that the dynamical regime of gauge theories that can be described via supergravity duals is not the usual one, where $x \ll 1$ at the UV cutoff. Nevertheless, it is believed that strongly-coupled gauge theories share some qualitative properties with their weakly coupled relatives; in particular, confinement and mass gap [6]. This belief has given rise to a blossoming of works where (super)gravity solutions are found, that describe higher-dimensional gauge theories compactified to 4-d [7], deformations of $\mathrm{N}=4$ 4-d supersymmetric gauge theories [8], and deformations of non-supersymmetric conformal theories [9, 10]. In latter example, the dual of the strongly-coupled gauge theory is a type 0, non-supersymmetric string theory.

A question posed by the existence of so many different ways of obtaining theories that are expected to have the same infrared behaviour is whether there exists a framework that allows to describe all the confining solutions at once. A slightly less ambitious objective is to find a framework that describes generically confining deformations of $\mathrm{N}=4$, 4-d super Yang-Mills.

In this paper, we describe such framework: 5-d gauged supergravity. We find a generic, universal solution of the equations of motion of gauged supergravity, that does not depend on the detailed form of its action. Our results exceed our expectations. Indeed, we find that, in our solution, the generic IR behaviour of the metric is universal, and agrees not only with all known confining solutions of type IIB supergravity, corresponding to softly broken $\mathrm{N}=4$ super Yang-Mills, but also with solutions of type 0 supergravity.

Our scenario has some similarities with the one proposed in [11, 12 for describing the universality class of confining strings, using five-dimensional non critical string theories. As a matter of fact our solution agrees in the IR with the one found in [11] for the simplest background with only the metric and the dilaton. It would be interesting to check if more complicated confining solutions, obtained by non-critical string theories, have the same IR behaviour as the one found in this paper.

We discuss the main features of our solution, as discreteness of the glueball mass spectrum and the existence of a mass gap. We present the computation of the string tensions and of the Wilson loop, and, in a concrete example, we show explicitly the area law for the quark-antiquark Wilson loop. We also find a general formula for condensates of scalar composite operators. This formula depends only on the near-singularity form of the metric. 
This paper is organised as follows. In Section 2, we describe the general model-independent results of our analysis. This Section describes the computation of the near-singularity metric, Wilson loop, glueball mass spectrum, and condensates. Section 3 applies the formalism of Section 2 to a specific example in 5 -d gauged supergravity. Section 4 contains our conclusions. Technical aspects of the computations in Sections 2 and 3 are confined to two Appendices.

\section{General Results}

The four-dimensional $\mathrm{N}=4 S U(N)$ super Yang-Mills theory is a superconformal field theory whose supergravity dual is type IIB supergravity compactified on $A d S_{5} \times S_{5}$ [2]. Type IIB supergravity contains all deformations of $\mathrm{N}=4$, with dimensions that remain finite in the large$x$ limit; they were found in [13]. Some of these deformations also exist in type IIB dimensionally reduced to five dimensions on $A d S_{5} \times S_{5}$, that is, in 5-d gauged $\mathrm{N}=8$ supergravity [14, 15].

Gauged N=8 supergravity has gauge group $S U(4)$ and 42 scalars. By the AdS/CFT correspondence, these scalars correspond to deformations of the $\mathrm{N}=4$ super Yang-Mills theory with dimension 2, 3, and 4. The scalar potential of 5-d gauged supergravity has several stationary points. All stationary points that satisfy the Breitenlohner-Freedman stability bound [16] correspond to local, unitary, four-dimensional conformal field theories. Some of these stationary points have been studied in [17, 18, 19].

It is widely believed that each solution of the equations of motion of 5-d gauged supergravity can be promoted to a solution of 10-d type IIB supergravity. Arguments in favour of this statement can be found in [17, 19, 20]; moreover, this is a theorem in the largely analogous case of 4-d gauged $S O(8)$ supergravity [21].

In a previous paper [17], we went beyond the study of conformal fixed points, and we found solutions of 5-d gauged supergravity that interpolate between two stationary points of the scalar potential. The 5-d metric of such solutions has two asymptotically- $A d S_{5}$ regions, describing the UV and IR conformal fixed points of the gauge theory.

In this paper, instead, we want to find solutions of 5-d gauged supergravity with only one asymptotically- $A d S_{5}$ region, corresponding to the UV N=4 super Yang-Mills $S U(N)$ theory. We will find that the IR behaviour of this metric is universal.

We are looking for a metric that preserves 4-d Poincaré invariance. This restricts its form to

$$
d s^{2}=d y^{2}+e^{2 \phi(y)} d x^{\mu} d x_{\mu}, \quad \mu=0,1,2,3 .
$$

We always work in the 5-d Einstein frame; $A d S_{5}$ with radius $R$ corresponds to $\phi=y / R+$ const. As in [17], we associate larger energies with increasing $y$. This means that we must look for solutions that approach $A d S_{5}$ when $y \rightarrow \infty$. 
We also allow for a $y$-dependence in some of the 42 scalars we have in our theory. The boundary conditions for the scalars is that they approach the $S O(6), \mathrm{N}=8$ invariant stationary point. This means that for $y \rightarrow \infty$ the dilaton is a constant, while all the other scalars vanish.

At large $y$, the behaviour of the scalars and the Einstein metric is complicated and nonuniversal, but their infrared behaviour is universal, for a wide class of solutions.

The 5-dimensional supergravity action for scalar fields coupled to the metric (11) reduces to

$$
L=\sqrt{-g}\left[\frac{R}{4}+\frac{1}{2} \sum_{a}\left(\partial_{y} \lambda^{a}\right)^{2}+V\left(\lambda^{a}\right)\right] .
$$

Here we have assumed canonical kinetic term for all scalars. This can always be achieved by an appropriate field redefinition. As we shall see shortly in our concrete example, the kinetic term is already canonical with a standard choice of variables. Einstein's equations and the equations of motion of the scalars following from (2) are:

$$
\partial_{y}^{2} \lambda^{a}+4 \partial_{y} \phi \partial_{y} \lambda^{a}=\frac{\partial V}{\partial \lambda^{a}}, \quad 6\left(\partial_{y} \phi\right)^{2}=\sum_{a}\left(\partial_{y} \lambda^{a}\right)^{2}-2 V
$$

Eqs. (3) have a universal runaway solution, independent of the detailed form of the potential. It is quite similar to the "singular instanton" solution found by Hawking and Turok [22]. Namely, let us assume that near $y=a$ the scalar fields and the metric diverge such that the potential term in Eqs. (3) is irrelevant. Then, one find a universal behaviour for both $\phi$ and $\lambda^{a}$ near $y=a:$

$$
\lambda^{a}=-K^{a} \log |y-a|+\text { constant }, \quad \phi=\frac{1}{4} \log |y-a|+\text { constant, } \quad \sum K^{a 2}=\frac{3}{8} .
$$

The metric, in particular, has a solution-independent behaviour:

$$
d s^{2}=d y^{2}+|y-a|^{1 / 2} d x^{\mu} d x_{\mu}
$$

For notational simplicity, we will put $a=0$ in the following.

The assumption that the potential is irrelevant is justified whenever $V$ is polynomial in $\lambda^{a}$. In gauged supergravity, typically, when scalars are canonically normalised, the potential is exponential in some of them. This means that the constants $K^{a}$ in Eq. (3) must obey some model-dependent inequalities.

Eq. (5) agrees with the behaviour of the 5-d Einstein-frame metric found in refs. [8, 10]. Agreement with ref. [8] is encouraging, since that paper studies a deformation of type IIB theory on $A d S_{5} \times S_{5}$; i.e. a model that falls into the class we are studying. More surprising is agreement with ref. [10]. After all, that paper studies a compactification of the non-supersymmetric type 0 superstring. Moreover, the form of the metric also agrees with that found in [11], using general arguments based on five-dimensional non-critical string theories, for a background with only the 
metric and the dilatonf. The common behaviour of the IR metric in all these different cases, type IIB, type 0 and non-critical strings, has a technical reason: all these models can be reduced to some effective theory for five-dimensional scalars. The fact that in all these theories one finds the same singularity in the metric may mean that the result we obtained is truly universal, and largely insensitive to the UV theory used to derive the IR confining behaviour.

We now discuss what are the characteristics of solutions that describe confining theories. The asymptotic behaviour in Eq. (5) does not completely specified the model. We must give the expressions for the tensions of the fundamental string and of the Dirichlet string. Since we made several Weyl rescaling with the purpose of reducing the ten-dimensional equations of motion in the string frame to the five-dimensional Einstein frame, we expect that the five-dimensional tensions depend on the supergravity scalars. These tensions are, in general, model-dependent. We compute below the tensions for a particular solution. However, from the computational details for the specific solution and the form of the five-dimensional supergravity, we expect a general power behaviour for the tensions. If we single out the particular scalar $\lambda^{0}$ as the five-dimensional dilaton, we have,

$$
T_{F 1}=y^{-\sum d_{a} K^{a}-K^{0}}, \quad T_{D 1}=y^{-\sum d_{a} K^{a}+K^{0}}
$$

where the coefficients $d_{a}$ depend on the specific model. This behaviour is in agreement with that found in ref. [8, 10]. Equipped with this result, we can study whether the theory confines by computing a Wilson loop, according to the original proposal in [23. The Wilson loop associated to a given boundary contour $C$ is obtained by minimising the classical action for a string free to move in the five-dimensional space but with endpoints on $C$. As reviewed in Appendix A, the knowledge of the asymptotic behaviour of the metric and the tensions is sufficient for extracting qualitative information about the Wilson loop. The result is that, in the range of the allowed values for $K^{a}$, it is possible to find different behaviours for the quark-antiquark potential, namely confinement as well as screening. However, confinement is generic for this class of solutions, in the sense that there is a large range of values of $K^{a}$ for which quarks confine and monopoles are screened. This is similar to what found in [10]. We exhibit below the detailed calculation in a specific example. Moreover, as we will see, even if the numerical details about the range of allowed values for $K^{a}$ are model-dependent, the physical picture is always the same. The asymptotic behaviour of the metric (5) conspires with the form of the tensions (6) to create a barrier for the fundamental string, which therefore cannot enter in the deep IR region. Since the string is forced to stay near to the boundary, an area law behaviour for the Wilson loop is expected [6].

\footnotetext{
${ }^{4}$ The relevant behaviour for this solution is determined by eq. (26) of [1]. Notice that we want to interpret $\phi=0$ not as the boundary, as in [11], but as the IR, as in the AdS-revised philosophy discussed in [12].
} 
Some comments about the validity of the solution are in order. Since the metric and the kinetic terms for scalars blow up in the IR, we expect that the supergravity solution is significantly corrected by the higher derivatives terms in the Lagrangian. This problem is common to all the proposed supergravity solutions for confining theories [8, 10]. We may expect that corrections are mildened by the fact that the Weyl tensor vanishes for our background [9]. Moreover, it was shown in [10], which contains solutions closely related to ours, that there exist particular values of the parameters for which the corrections are suppressed. In the best of possible worlds, the corrections will simply slightly change the range of parameters, and we saw that the physics of our solution is not sensitive to such details. Moreover, confinement is obtained when the fundamental string does not probe the far IR region, whose details become therefore irrelevant. It is possible then that the particles wave-functions and the fundamental string world-sheet, needed for computing the mass spectrum and the Wilson loops, always live in a region where curvature and higher derivatives terms are still under control.

We now discuss the existence of a mass gap and the glueball spectrum. This is done as in [6] by looking for normalizable solutions of the equation of motion of a minimally coupled massless scalar in the metric (1) The action of the scalar is

$$
S=\int_{0}^{\infty} d y\left(e^{4 \phi}\left|\partial_{y} \psi\right|^{2}+k^{2} e^{2 \phi}|\psi|^{2}\right), \quad k^{2}=k^{\mu} k_{\mu}
$$

Integrating by part Eq. (17), and using the equation of motion and $e^{4 \phi}=0$ at $y=0$ we find $S=0$ for the normalizable solution, which approaches a constant at $y=0$. Therefore, $k^{2}$ must be strictly negative, proving the existence of a mass gap. Discreteness of the spectrum follows from requiring normalizability at large $y$ [6].

The equation of motion derived from action (7) is

$$
-\partial_{y}^{2} \psi-4 \partial_{y} \phi \partial_{y} \psi+k^{2} e^{-2 \phi} \psi=0
$$

The spectrum of glueballs can be studied using a WKB approximation 24. Standard calculations lead to the following form for the WKB wave function:

$$
\begin{aligned}
& \psi(y)=C[-2 \phi(y) \pm i p(y)]^{-1 / 2} e^{-2 \phi(y) \pm i \int_{a}^{y} d y p(y)}, \quad 0<y<b \\
& \psi(y)=C^{\prime}[2 \phi(y)+|p(y)|]^{-1 / 2} e^{-2 \phi(y)-\int_{y}^{a} d y|p(y)|}, \quad y<a \\
& \psi(y)=C^{\prime \prime}[2 \phi(y)+|p(y)|]^{-1 / 2} e^{-2 \phi(y)-\int_{b}^{y} d y|p(y)|}, \quad y>b .
\end{aligned}
$$

Here, $p(y)=\sqrt{-k^{2} e^{-2 \phi(y)}-4 \phi_{y}^{2}(y)}, C, C^{\prime}, C^{\prime \prime}$ are constants, and $a, b$ are the classical turning points, $p(a)=p(b)=0$. The existence of these points follow from the form of $p(y)$ at small and large $y$. At small $y$, it is:

$$
p(y)=-k^{2} y^{-1 / 2}-\frac{1}{4} y^{-2}, \quad y \ll 1
$$


Notice that this expression is universal, since it depends only on the near-singularity metric in Eq. (5). At large $y, p(y)$ is also universal, since there the metric approaches the AdS form: $\phi(y)=y$; thus,

$$
p(y)=-k^{2} e^{-2 y}-4
$$

At $y=0, p(y)$ diverges to $-\infty$; at intermediate $y, p(y)$ is positive when $k^{2}<0$; at large $y, p(y)$ becomes negative again. This proves the existence of turning points. As usual in the case of a bound classical trajectory, the bound state spectrum is discrete. In the WKB approximation, it is given by the Bohr-Sommerfeld quantisation rule [25]

$$
\int_{a}^{b} d y \sqrt{E_{n} e^{-2 \phi(y)}-4 \phi_{y}^{2}(y)}=\pi(n+\alpha), \quad E_{n}=-k^{2}
$$

with $\alpha$ a constant of order one. Eq. (14) is the starting point for numerical and analytical studies of the glueball spectrum. In this paper, though, we do not want to join this thriving cottage industry; instead, we want to exhibit general, model independent, features of supergravity duals of gauge theories. Thus, we shall abandon the study of the glueball spectrum, and discuss another model-independent feature of our solution, namely, the existence of condensates.

The condensate of a field theoretical operator $O_{a}$ that couples to the source $\lambda^{a}$ reads [3, 4]:

$$
\left\langle O_{a}\right\rangle=\frac{\delta S_{5 d}}{\delta \lambda^{a}}
$$

Here $S_{5 d}$ is the action of 5 -d gauged supergravity, computed on the solution of the equations of motion (3). Thanks to the equations of motion, the right hand side of Eq. (15) is a total derivative:

$$
\left\langle O_{a}\right\rangle=\left[\frac{1}{2} e^{4 \phi(y)} M_{a b}(\lambda) \lambda_{y}^{b}\right]_{0}^{\infty} .
$$

Unlike in Eq. (3), here we allow for a non-canonical kinetic term. Whenever the potential $V$ can be neglected, the universal runaway solution in Eq. (4) still holds. Near $y=0$ it reads

$$
e^{4 \phi(y)} M_{a b}(\lambda) \lambda_{y}^{b}=\text { constant } \equiv-K_{a} .
$$

In Eq. (16) the upper boundary diverges at $y=\infty$ and must be subtracted. This UV regularization is determined by requiring that condensates vanish for geometries with two asymptotic AdS regions. In that case, the coordinate $y$ runs from $y=-\infty$ to $y=+\infty$, and the contribution of the lower boundary to Eq. (16) vanishes, while the contribution of the upper boundary is still divergent. After UV regularization, Eq. (16) reduces to a very simple form:

$$
\left\langle O_{a}\right\rangle=\frac{1}{2} K_{a} .
$$

Notice that this formula for the condensate is independent of all the fine details of the metric, in particular, its behaviour at large $y$. Moreover, it is finite without any ad-hoc IR regularization, and also gives a physical meaning to the constants of integration $K_{a}$. 


\section{An Example}

Let us apply now the machinery developed in the previous Section to a specific example. A simplified theory with only two scalars it is already enough to describe the general behaviour of confining theories, and allows us to make contact with the solutions found in [8, 10].

It is easy to make contact with the solution found in [8] . We are studying the same problem in the dimensionally reduced theory. The solution in [8] corresponds to a single running field, the five-dimensional dilaton, which has zero potential [15]. We therefore expect an IR metric as in Eq. (5). A simple Weyl rescaling indeed shows that the solution in [8] has the IR behaviour given by Eq. (5). The tensions also agree with the discussion in the previous Section.

More general ten-dimensional solutions, corresponding to perturbations of $A d S_{5} \times S^{5}$ with some other fields, and breaking the $S U(4)$ invariance, have not yet been found. These solutions are quite natural to consider, since most of them correspond to perturbations of super $\mathrm{N}=4$ Yang-Mills with mass terms for scalars and fermions. The technical difficulty of finding a full ten-dimensional solution can be easily overcome if we are interested in the less complete but still quite interesting corresponding five-dimensional solution. In ten dimensions, $S U(4)$ noninvariant fields are automatically sources for the dilaton, which then starts to run. We can mimic this solution in five dimensions by considering the above equations of motions for two scalars, the dilaton (which does not appear in the potential) and a scalar with a non-trivial potential. We consider, for simplicity, the lucky case in which all other scalars can be consistently set to zero. The solution in Eq. (四) depends on two constants, $K$ and $K_{0}$, with the constraint $K^{2}+K_{0}^{2}=3 / 8$. A potential for $\lambda$, which in supergravity generally is of the asymptotic form $e^{c \lambda}$, is irrelevant if and only if

$$
0<c K<2, \quad K^{2}+K_{0}^{2}=\frac{3}{8} .
$$

Generically, this equation can be satisfied for certain values of $K$, in which case the IR metric assumes the form given in Eq. (5). We could also try to consider a solution with a fixed value of the dilaton. In this case, it is not obvious that the potential is irrelevant; it must happen that

$c \sqrt{\frac{3}{8}}<2$ and, as we will see, this is not the case, in general.

The solutions found in [10] do not obviously fall in the class of examples we are considering; indeed, they are not obtained from an $\mathrm{N}=4 \mathrm{UV}$ fixed point. The source for the dilaton is, instead, a non-zero tachyon field, which exists only in type 0 theories. However, the type 0 equations of motions also reduce to a certain Lagrangian for a set of scalars with an effective potential. Under these circumstances, it is not surprising that the solution found in [10] has the same generic IR behaviour as our solution.

To study a concrete example, let us consider a deformation of the $\mathrm{N}=4$ Yang-Mills theory that corresponds, in $\mathrm{N}=1$ notations, to a mass term for the three chiral multiplets $X_{i}$. The 
theory flows in the IR to pure $\mathrm{N}=1$ Yang-Mills, which confines. To obtain the standard $\mathrm{N}=1$ pure Yang-Mills with fixed scale $\Lambda$ we need a fine tuning of the UV parameters, in which the mass $m$ diverges while the t'Hooft coupling constant, $x$, goes to zero as a logarithm of $m$. This is outside the regime of validity of supergravity, which requires a large $x$, but we may still expect to see from supergravity the qualitative properties of the theory, as, for instance, confinement. The supergravity mode corresponding to a mass term for the chiral multiplets was identified in [26]. The 42 scalars of the five-dimensional supergravity transform as $\underline{1}, \underline{20}, \underline{10}$ under $S U(4)$. The explicit form of the CFT operators to which these supergravity fields couple can be found, for example, in [17]. The mass term for the chiral multiplets appears in the decomposition $\underline{10} \rightarrow \underline{1}+\underline{6}+\underline{3}$ of $S U(4)$ under $S U(3) \times U(1)$. A non-zero vev for the $S U(3)$ singlet contained in this decomposition was studied in [17, 18]. It corresponds to a deformation of $\mathrm{N}=4$ super Yang-Mills that leads to a non-supersymmetric, conformal IR fixed point. A non-zero vev for $\underline{6}$, when represented as a complex symmetric matrix, $m_{i j}$, corresponds to the $\mathrm{N}=1$ supersymmetric mass deformation $\int d^{2} \theta m_{i j} X_{i} X_{j}+$ c.c.. Since we want to compute and to control the asymptotic behaviour of the potential, we need to reduce the number of scalars which we turn on. If we are not careful, a non-zero vev for the scalar $m$ will induce non-zero vevs for other scalars as well, due to the existence of linear couplings of $m$ to the other fields in the potential. This is indeed the case for generic $m_{i j}$. However, if we further impose $S O(3)$ symmetry, by taking an $m_{i j}$ proportional to the identity matrix, a simple group theory exercise shows that all the remaining fields can be consistently set to zero.

The five-dimensional action of the scalars, given in ref. [14], is written in terms of a $27 \times 27$ matrix $U$, transforming in the fundamental representation of $E_{6}$ and parametrising the coset $E_{6} / U S p(8) . U$ can be written as $U=e^{X}, X=\sum_{a} \lambda_{a} T_{a}$, where, in a unitary gauge, $T_{a}$ are the generators of $E_{6}$ that do not belong to $U S p(8)$. This matrix has exactly 42 real independent parameters, which are the scalars of the supergravity theory. The Lagrangian has the form

$$
L=\sqrt{-g}\left[-\frac{R}{4}-\frac{1}{24} \operatorname{Tr}\left(U^{-1} \partial U\right)^{2}+V(U)\right] .
$$

Since the rules for computing the potential were described in [15], and recently reviewed and applied in [18, 19], here we will recall only the main points of the computation. First, one chooses a convenient parametrisation for the coset manifold representative which makes the kinetic terms canonical. A possible parametrisation, suitable for studying the breaking $S U(4) \rightarrow$ $S U(3) \times U(1)$, has been extensively described in [18]. Secondly, one performs some gammamatrix algebra. Conventions, details and computational tricks can be found in ref. [15]. A summary of the relevant formulae can be found in Appendix B. The result for the diagonal scalar $m \delta_{i j}$ is

$$
L=\sqrt{-g}\left[-\frac{R}{4}+\frac{1}{2}(\partial m)^{2}-\frac{3}{8 R^{2}}\left(\cosh ^{2}(2 m)+7\right)\right]
$$


where $R$ is the curvature of $A d S_{5}$. The above potential passes two crucial checks: when expanded for small $m$ it reproduces the cosmological constant of the $S U(4)$ symmetric AdS-vacuum, and the mass for the scalar $m$-which is, according to [13], $-3 / R^{2}$, the right value to be the source of a dimension-three operator in the CFT. In any case, the asymptotic behaviour of the potential can be figured out even without a detailed computation. With only one scalar and the above form for $U=e^{m T}$, where $T$ is a particular generator of $E_{6}$, suitably normalised, the kinetic term is automatically canonical. Moreover, the potential is quartic in $U$ making quite plausible the asymptotic behaviour $\sim e^{4 m}$.

If we turn on only the field $m$, and implement the constraint in Eq. (19), we easily discover that the potential cannot be neglected, and, therefore, the solution in eq. (3) does not describe well the IR physics. This is due to the fact that the power of $e^{m}$ in the potential is too large. As we already discussed, this problem can be easily circumvented by turning on other scalars. The natural choice is to turn on the dilaton. We will call $\rho$ the five-dimensional dilaton. It has a canonical kinetic term $\frac{1}{2}(\partial \rho)^{2}$, and it does not appear in the potential. It is not obvious whether the five-dimensional solution preserves $\mathrm{N}=1$ supersymmetry, as one may naively think. The solution with only the dilaton was argued to break all supersymmetries in [8]. It is possible that some particular solution with non-zero fields $m$ and $\rho$ preserves $\mathrm{N}=1$ supersymmetry; the safe way to check this is to look at the supersymmetry variations of the fermions. However, here we are not really interested in an $\mathrm{N}=1$ solution. Rather, we want to prove that for a generic, not necessarily supersymmetric solution, the IR behaviour is universal, and that confinement is expected for a large range of values of the parameters $K_{a}$, without requiring fine tuning. The non-zero dilaton induces some other $S O(6)$ invariant deformations of the $\mathrm{N}=4 \mathrm{UV}$ fixed point. Beside the obvious $F_{\mu \nu}^{2}$ term, the only other $S O(6)$-invariant supergravity field that may appear is $F^{4}$. This is however irrelevant at the UV fixed point and does not explain the solution in [8]. It was argued in the second paper in ref. [8] that the usually neglected nonchiral operators, like the diagonal mass term for the six scalars of the $\mathrm{N}=4$ theory, may be also induced as deformations of the $\mathrm{N}=4 \mathrm{UV}$ fixed point by the running dilaton solution, and that they may play a role in the field theory interpretation, despite their infinite anomalous dimension. Whether or not we accept this interpretation, the solution with non-zero dilaton and non-zero scalar $m$ is reasonably associated to a deformation of $N=4$ super Yang-Mills, that renders massive at least all the scalars and fermions in the chiral multiplets. In a genuine $\mathrm{N}=1$ theory, the gaugino will remain massless to give pure $\mathrm{N}=1$ Yang-Mills. In a non supersymmetric

\footnotetext{
${ }^{5}$ With a deformation of the form $\int d^{2} \theta m X_{i} X_{i}+$ c.c., we find in the Lagrangian also a diagonal mass term for the six scalars of the $\mathrm{N}=4$ theory, quadratic in the parameter $m$, and, therefore, not seen in a linearization in $m$. The CFT operator associated to the supergravity scalar $m$ is obtained by linearizing around the $\operatorname{AdS}_{5} \times S^{5}$ solution, and it will never see the quadratic term. The appearance of this non-chiral operator at the second order may be an hint for the inclusion of stringy state, or simply due to an operator mixing.
} 
theory it will probably become massive. In any case, we reasonably expect a confining theory in the IR.

To prove confinement we need to compute the Wilson loop and show that it exhibits an area law. We first need to compute the string tension in five dimensions. The tension of the fundamental string (or the D1-string) can be read from the coefficient of the kinetic term for the NS-NS (or R-R) antisymmetric tensor in the ten dimensional Lagrangian in the Einstein frame,

$$
\frac{1}{T_{F 1}^{2}} H_{N S-N S}^{2}+\frac{1}{T_{D 1}^{2}} H_{R-R}^{2} .
$$

A simple Weyl rescaling shows that this property is valid also in the five-dimensional theory in the Einstein frame. We need, therefore, the kinetic terms of the antisymmetric tensors in the five-dimensional supergravity. The five-dimensional Lagrangian has a global $S L(2 ; R)$ symmetry, which helps in identifying the various types of strings. There are 12 antisymmetric tensors $B_{\mu \nu}^{I \alpha}, I=1, \ldots, 6, \alpha=1,2$, transforming in the $\underline{6}$ of $S U(4)$, and in the two-dimensional representation of $S L(2 ; R)$. We interpret the index $I$ as signalling that our five-dimensional string remembers its ten-dimensional origin. The position of the string on the five-sphere, indeed, must be specified in a bona-fide ten-dimensional computation. We will consider the case in which the kinetic terms for $B^{I \alpha}$ are diagonal in $I$. The second index, $\alpha$, specifies a whole multiplet of strings transforming under $S L(2 ; R)$, as it is expected from the S-duality of $\mathrm{N}=4$ super Yang-Mills. The antisymmetric-tensor kinetic term was written in [15] in a first order formalism:

$$
\epsilon_{\alpha \beta} B_{I \alpha} \wedge d B_{I \beta}+A_{I \alpha, J \beta} B_{I \alpha} \wedge * B_{J \beta} .
$$

$A_{I \alpha, J \beta}$ is a symmetric matrix which depends on the scalars and whose explicit expression is given in Appendix B.

The problem of writing down the BPS formula, which relates the tension of the strings to their charges, is non trivial, especially in a gauged supergravity, where the fields $B_{I \alpha}$ are massive. In this paper we are taking the quite plausible point of view of believing that the five-dimensional $S L(2 ; R)$ is the same as the ten-dimensional one and it is broken to $S L(2 ; Z)$ by non-perturbative effects. We also trust naive dimensional reduction arguments from ten dimensions 巳. For a diagonal matrix $A$ we simply have

$$
B_{1} \wedge d B_{2}+a_{1}\left|B_{1}\right|^{2}+a_{2}\left|B_{2}\right|^{2}
$$

Now, if we choose one of the fields (say $B_{1}$ ) and solve the equation of motion of $B_{2}$, we can recover

\footnotetext{
${ }^{6}$ The general discussion in the previous section would not be affected by the discovery of the naïvety of this argument. And, for the particular example we are discussing, only few details would change; certainly not the general conclusions.
} 
the standard action for a massive antisymmetric tensor, which is expected in KK reduction [13]:

$$
\frac{1}{a_{2}}\left|d B_{1}\right|^{2}+m\left|B_{1}\right|^{2}
$$

When $A$ is not diagonal, one must be careful in going from the first order formalism to the second order one. One can easily see that the square-root of the eigenvalues of the matrix $A_{I \alpha, J \alpha}$, restricted to diagonal $S L(2, R)$ indices, gives the tensions of the strings. In the parametrisation used in this paper, the square of the tension of the fundamental string is given by an eigenvalue of $A_{I 1 J 1}$, while the square of the tension of the D1-string is given by an eigenvalue of $A_{I 2, J 2}$. The matrix $A$ is essentially the square of the matrix $U$; the precise formula is given in Appendix B. While the potential is independent of the dilaton, $A$ crucially depends on it. It is easy to compute the eigenvalues of $A$ for the maximally supersymmetric vacuum corresponding to $\mathrm{N}=4$ super Yang-Mills, when all the scalars but the dilaton are zero. The detailed computation can be found in Appendix B. The not surprising result is that the two eigenvalues of $A,\left(a_{1}, a_{2}\right)$, are exactly $\left(e^{2 \rho}, e^{-2 \rho}\right)$. The tensions can be computed without difficulty even when other scalars are non-zero. What one finds is that, due to the quadratic dependence on $U$, the contribution of $m$ to both tensions has the asymptotic form $\sim e^{m}$. This result is general enough, and predicts tensions of the form $\left(e^{(c / 4) \lambda+\rho}, e^{(c / 4) \lambda-\rho}\right)$, where $c$ is the (integer) constant appearing in the asymptotic behaviour of the potential $\sim e^{c \lambda}$.

We now summarise the characteristics of the model. First, we see that the potential behaves as $e^{4 m}$. The allowed range of parameters, given by Eq. (19), is

$$
0<K<\frac{1}{2}, K^{2}+K_{0}^{2}=\frac{3}{8} .
$$

The IR behaviour of the metric is as in Eq. (5), and the tensions for the fundamental and the D1 strings are:

$$
T_{F 1}=e^{m+\rho}=y^{-K-K_{0}} ; \quad T_{D 1}=e^{m-\rho}=y^{-K+K_{0}} .
$$

We are now ready to compute the Wilson loop. The computation closely parallels that in ref. [23]. The world-sheet action for a fundamental (or D1-) string in the background (11) is

$$
S=\int d \tau d \sigma \sqrt{G_{i n d}}=T \int d x T(y) e^{\phi(y)} \sqrt{\left(\partial_{x} y\right)^{2}+e^{2 \phi(y)}}
$$

The Wilson loop is obtained by minimising this action of a string whose endpoints span a rectangle on the boundary of the space, with one side of length $L$ in the direction $x$, and another one of length $T$ along the time axis. We choose the standard embedding $\sigma=x$ and factorize the trivial integration in time. It is useful to change coordinates in such a way that the quark-antiquark (or monopole-antimonopole) energy is

$$
E=S / T=\int d x \sqrt{\left(\partial_{x} u\right)^{2}+f(u)} .
$$


The change of variable and the function $f(u)$ are given by

$$
\frac{\partial u}{\partial y}=T(y) e^{\phi(y)}, \quad f(u)=T^{2}(u) e^{4 \phi(u)} .
$$

The computation of the Wilson loop for generic metrics has been widely discussed in the literature [23, 27, 28, 10]. Here we give a short review of what is expected about the Wilson loop behaviour, once the function $f(u)$ has been specified (more details can be found in Appendix A). The boundary of the space is $u=+\infty$ and the asymptotic behaviour of $f(u)$ near the boundary, $f(u) \sim u^{4}$, is obtained from the $A d S_{5}$ UV metric. The metric can be regular in the IR region, or it can extend only up to some point $u=a$, if there is a horizon or, as in our case, if the metric has a singularity. For notational simplicity we consider $a=0$. If the function $f(u)$ has a minimum at a finite point, $\bar{u}$, the fundamental string will then find energetically favourable to end at $\bar{u}$ without entering in the deep IR region. Under these circumstances, we expect an area law for the Wilson loop [0, [10]. If instead the function $f(u)$ is monotonic in the IR, different behaviours may be expected; they are reviewed in Appendix A. It may happen that $f(u)$ behaves as $u^{4}$ both in the UV and in the IR, but with different coefficients. This is the case for the RG flow between different fixed points considered in [17, 18]. Another interesting possibility, which will be very important for us, is the behaviour $f(u) \sim u^{\gamma}, 0<\gamma<2$. In this case the string goes straight to the point $u=0$, continues for a distance $L$ along $x$, and returns back to $u=\infty$. Since $f(0)=0$, it does not cost any energy to separate the quark at $u=0$. This corresponds to electric (or magnetic) screening [27, 28].

Let us apply these results to our solution. Using the coordinates in Eq. (30), we find for the function $f(u)$, in the case of a pair of quarks and a pairs of monopoles, respectively,

$$
f_{q \bar{q}}(u) \sim u^{\frac{1-2\left(K+K_{0}\right)}{5 / 4-\left(K+K_{0}\right)}}, \quad f_{m \bar{m}}(u) \sim u^{\frac{1-2\left(K-K_{0}\right)}{5 / 4-\left(K-K_{0}\right)}} .
$$

It is easy to check that, in the allowed range of values for the constants $K$ and $K_{0}$, given in Eq. (26)], $f_{q \bar{q}}$ diverges while $f_{m \bar{m}} \sim u^{\gamma}$ with $0<\gamma<2$. This means that, in our solution, the theory confines for all values of $K$ and $K_{0}$. There is a linear potential for quarks, and monopoles are screened. Since confinement is obtained when the fundamental string does not probe the far IR region, most of the details of the solution are therefore irrelevant. Provided that the function $f(u)$ diverges in the IR, no matter how, confinement is guaranteed.

The form of the solution we found is quite similar to that found in $\left[10 \|^{\text {. }}\right.$. A difference with

\footnotetext{
${ }^{7}$ We are taking $K_{0}>0$. The case $K_{0}<0$ is clearly related to the one discussed here by an S-duality.

${ }^{8} \mathrm{We}$ can easily make contact with the generic IR solution in [10]. It depends on three parameters subject to a constraint. Upon reduction to five dimensions, the metric has, indeed, the form of Eq. (5), and does not depend on any parameter. Two parameters are needed to specify the tensions of the fundamental string and the Dirichlet string. The relation between our parameters and those in 10$]$ is $K=5 \frac{\alpha_{1} / 2-\alpha_{2}}{\alpha_{1}-5 \alpha_{2}}, K_{0}=-\frac{\alpha_{0}}{\alpha_{1}-5 \alpha_{2}}$. With this parametrisation, the quadratic constraint that we find is slightly different from the one in [10]. This is not surprising since we are dealing with type IIB and ref. 110] with type 0. We are claiming that the form of the solution is universal; certainly details are not.
} 
ref. [10] is that we find confinement for all values of the parameters. This is expected from the physical interpretation as a mass deformation of $\mathrm{N}=4$ super Yang-Mills. More general solutions, as the one in [10], for instance, which exhibit electric confinement or screening according to different values for the parameters, can be easily accommodated by considering more scalars or a different potential.

\section{Conclusions}

In this paper, we have found a very satisfying and novel form of infrared universality for supergravity duals of strongly coupled gauge theories. Specifically, we have found that, generically, there exists a runaway solution of the coupled scalar-Einstein equations of motion of 5-d type IIB gauged supergravity. Gauged 5-d supergravity is a convenient and general framework to study deformations of $\mathrm{N}=4$ super Yang-Mills theories in the limit of large 't Hooft parameter. The runaway solution we described is universal and independent of the detailed form of the supergravity action. It gives a universal (singular), 5-d metric and universal formulae for the string tensions and scalar condensates. This universality explains why many examples of confining duals of 4-d gauge theories share common properties. It is also encouraging in that it may mean that even for small 't Hooft parameter, some generic properties of the supergravity approximation to gauge theories, as confinement and mass gap, can survive string corrections.

\section{Acknowledgements}

M. Petrini would like to thank A. A. Tseytlin for discussions. L. Girardello and M. Petrini are partially supported by the European Commission TMR program ERBFMRX-CT96-0045, wherein L. Girardello is associated to the University of Torino, and M. Petrini to the Imperial College, London. L. Girardello is also supported in part by INFN and MURST. M. Porrati is supported in part by NSF grant no. PHY-9722083.

\section{Appendix A: Generalities About the Wilson Loop}

In this Appendix we review the Wilson loop calculation of refs. [23, 27, 28, 10].

We use coordinates in which the energy of a pair of heavy quark is

$$
E=S / T=\int d x \sqrt{\left(\partial_{x} u\right)^{2}+f(u)}
$$

In the $A d S_{5}$ case, we have $f(u)=u^{4}$. The $A d S_{5}$ boundary is at $u=\infty$, while the singularity is at $u=0$. The Wilson loop is obtained by minimising the corresponding action for a string world-sheet which has a boundary at $u=\infty$. We can take a rectangle at the boundary of the 
space, with one side of length $L$ in the direction $x$ and another one of length $T$ along the time axis. We choose the standard embedding $\sigma=x$, and we factorize the trivial integration in time.

In the conformal $A d S_{5}$ case, the Wilson loop is not proportional to the boundary area since the function $f(u)$ is much smaller inside $A d S_{5}$ and it is energetically favourable for the string to go inside $A d S_{5}$. If, for any reason -such as a horizon, which introduces a cutoff, as is the case of theories at finite temperature, or a barrier- the string cannot extend far enough from the boundary, we expect an area law and confinement.

Consider now a general metric corresponding to a UV non-trivial fixed point. The boundary of the space is $u=+\infty$ and the asymptotic behaviour of the function $f(u) \sim u^{4}$ near the boundary is obtained from the $A d S_{5} \mathrm{UV}$ metric. The metric may be regular in the IR region or extend only up to some point $u=a$ if there is a horizon or, as in our case, if the metric has a singularity. For notational simplicity we consider $a=0$.

The action in Eq. (A.1) can be minimised as in [23]. Defining $u_{0}$ as the turning point of the string, we have, exactly as in [23], two equations which implicitly give $E$ as a function of $L$ :

$$
\begin{aligned}
\frac{L}{2} & =\int_{u_{0}}^{\infty} \frac{d u}{\sqrt{\frac{f(u)}{f\left(u_{0}\right)}\left(f(u)-f\left(u_{0}\right)\right)}}, \\
E & =\int_{u_{0}}^{\infty} d u\left(\frac{\sqrt{f(u)}}{\sqrt{f(u)-f\left(u_{0}\right)}}-1\right)-1 .
\end{aligned}
$$

The derivation of these equations closely parallels that in ref. [23], and it will not be repeated here. The divergent expression for the energy has been regularized as in [23] by subtracting the infinite contribution due to the mass of the heavy quarks.

This implicit equation for $E(L)$ is hard to solve analytically. However, the main contribution to the energy usually comes from the region around $u_{0}$. The contribution from the large- $u$ region in the integral, where $f(u) \sim u^{4}$, is always subtracted, since it diverges.

Consider first the case in which $f(u)$ is monotonic. Under the assumption that the integrals are dominated by the region around $u_{0}$, we get

$$
L \sim \sqrt{\frac{u_{0}}{f^{\prime}\left(u_{0}\right)}}, E \sim \sqrt{\frac{u_{0} f\left(u_{0}\right)}{f^{\prime}\left(u_{0}\right)}} .
$$

It may happen that $f(u)$ behaves as $u^{4}$ both in the UV and in the IR, but with different coefficients. This is the case for the RG flow between different fixed points considered in [17, 18]. In this case, for $L \rightarrow \infty, u_{0} \sim 1 / L$ approaches the far IR region. The integrals are dominated by the IR, which is still an $A d S_{5}$ space with a different radius. We are back to the computation in [23] with a different radius for the space. We obviously find that, for $L \rightarrow \infty, E \sim u_{0} \sim 1 / L$, as expected in a conformal theory. 
It is interesting to consider the case in which $f(u) \sim u^{\gamma}, 0<\gamma<2$. Since $L \sim u_{0}^{1-\gamma / 2}$, we see that for small $u_{0} L$ goes to zero. $L$ goes to zero also for large $u_{0}$, since, due to the UV asymptotic form of $f(u)$, the integral in Eq. (A.2) is always convergent for $u \rightarrow \infty$. As a consequence, $L$ is a function of $u_{0}$ bounded from above. At first sight, there is no way to probe the IR behaviour corresponding to $L \rightarrow \infty$. However, it was argued in [27, 28] that for large enough $L$ a different physical picture takes over. For large $L$, the string will go to the point $u=0$ along a straight line, proceed for a distance $L$ along $x$ and return to $u=\infty$. Since $f(0)=0$, it does not cost any energy to separate the quarks at $u=0$. This corresponds to electric (or magnetic) screening [27, 28].

Consider now the case that $f(u)$ is still monotonic but does not vanish in the IR. If $f(u) \geq$ const $>0$, the integral in Eq. (A.1) is certainly greater than $L$ and we expect an area law behaviour for the Wilson loop [6]. This is the case, for example, of theories at finite temperature, where there is a horizon at a finite $u_{T}$. Since $u>u_{T}$, the string cannot extend far enough from the boundary. In this case, we expect that the Wilson loop will always grow at least as the area [6]. This is indeed confirmed by the expression $f \sim$ const $+\left(u-u_{T}\right)^{2}$ [27, 28]. We see that $u_{0}$ will approach the horizon.

Beside the existence of a horizon, there is a second mechanism that prevents the string from going far enough from the boundary. If the function $f(u)$ diverges near $u=0$, it will create a barrier, giving rise to a confining behaviour for the Wilson loop. $f(u)$ will have a minimum at a finite point $\bar{u}$. Assuming $f(\bar{u})>0$, we have that $f(u) \geq f(\bar{u})>0$. The fundamental string will then find energetically favourable to end at $\bar{u}$ without entering in the far IR region. Under these circumstances, we expect an area law for the Wilson loop [6, 10]. We can explicitly check the behaviour of the Wilson loop. $L$ automatically diverges if $u_{0}$ approaches $\bar{u}$. Suppose that $f^{\prime}(\bar{u})=0, f^{\prime \prime}(\bar{u}) \neq 0$-the case in which the first non-zero derivative is the $k$-th one is completely analogous. From Eqs. (A.2) we have:

$$
\frac{L}{2} \sim \operatorname{const} \int_{u_{0}}^{\infty} \frac{d u}{u-\bar{u}} \rightarrow \infty, E \sim \text { const } \int_{u_{0}}^{\infty} \frac{d u}{u-\bar{u}} \sim L
$$

and we see that, generically, $E \sim L$.

\section{Appendix B: Conventions and Useful Formulae for the Lagrangian}

In this Appendix we give the relevant formulae needed to compute the Lagrangian in Eq. (21). Since the tools for computing the potential have been already described in details in [15] and recently reviewed in [18, 19], we do not aim to be self-contained but just to sketch the outline of the computation. The reader may refer to the papers mentioned above for more details. 
Ref. [19], in particular, gives a nice short review of the philosophy behind these computations.

The five-dimensional Lagrangian for the scalars is written in terms of the $27 \times 27$ matrix $U$ parametrising the coset $E_{6} / U S p(8)$

$$
L=\sqrt{-g}\left[\frac{R}{4}-\frac{1}{24} \operatorname{Tr}\left(U^{-1} \partial U\right)^{2}+V(U)\right] .
$$

In a unitary gauge, $U$ can be written as

$$
U=\exp X
$$

where $X=\sum_{a} \lambda_{a} T_{a}$ is given by the 42 generators of $E_{6}$ that do not belong to $U S p(8)$ : these 42 independent parameters correspond to the 42 scalars. The precise form of the generator corresponding to a given scalar can be worked out using the global and local symmetries of the problem, and the fact that $U$ maps an element of the representation $\underline{27}$ of $E_{6}$ into itself. More precisely, one has to remember that only the group $S U(4) \times S L(2 ; R)$ is a symmetry of the Lagrangian. The 42 scalars then decompose according to

$$
42 \rightarrow 20_{(0)}^{\prime}+10_{(-2)}+\overline{10}_{(2)}+1_{(4)}+1_{(-4)}
$$

while the vectors in the $\underline{27}$ decompose as

$$
27 \rightarrow 15_{(0)}+6_{(2)}+6_{(-2)} .
$$

The subscripts denote the charges of the $U(1)$ factor in $S L(2 ; R)$.

The general parametrisation for $U$ is given by Eqs. (A.36) of [15], with the following conventions for the indices: the $\underline{27}$ of $E_{6}$ is represented by a couple of antisymmetric symplectic-traceless indices $A, B$, running form 1 to 8 , and, in the $S U(4) \times S L(2 ; R)$ basis, it decomposes as

$$
\begin{aligned}
27 & \rightarrow 15+(6,2) \\
{[A B] } & \rightarrow([I J], I \alpha)
\end{aligned}
$$

with $I, J=1, \ldots, 6$ indices of $S U(4)$ and $\alpha=1,2$ indices of $S L(2 ; R)$. The brackets mean that the indices are antisymmetrized.

If one is interested, as we are, in breaking the $S U(4)$ factor to $S U(3) \times U(1)$, a more suitable parametrisation for $U$ is given in [18], with the following basis for the $\underline{27}$ :

$$
27 \rightarrow\left(1_{(0,0)}, 3_{(4,0)}, \overline{3}_{(-4,0)}, 8_{(0,0)}, 3_{(-2,2)}, \overline{3}_{(2,2)}, 3_{(-2,-2)}, \overline{3}_{(2,-2)}\right)
$$

This time the subscripts indicate the charges under the $U(1) \subset S U(4)$ and the $U(1) \subset S L(2 ; R)$, respectively. 
In our example, we turn on the dilaton field, $\rho$, and the scalars $m$ in the $\underline{6}_{(2,-2)}$ of $S U(4)$. From ref. [18], the matrix $U$ has the form

$$
U=e^{X_{0}} e^{X}
$$

where

$$
X_{0}=\left(\begin{array}{cccccccc}
0 & 0 & 0 & 0 & 0 & 0 & 0 & 0 \\
0 & 0 & 0 & 0 & 0 & 0 & 0 & 0 \\
0 & 0 & 0 & 0 & 0 & 0 & 0 & 0 \\
0 & 0 & 0 & 0 & 0 & 0 & 0 & 0 \\
0 & 0 & 0 & 0 & 0 & 0 & \rho e^{i \alpha} & 0 \\
0 & 0 & 0 & 0 & 0 & 0 & 0 & \rho e^{i \alpha} \\
0 & 0 & 0 & 0 & \rho e^{-i \alpha} & 0 & 0 & 0 \\
0 & 0 & 0 & 0 & 0 & \rho e^{-i \alpha} & 0 & 0
\end{array}\right), \quad X=\left(\begin{array}{cccccccc}
0 & 0 & 0 & 0 & 0 & 0 & 0 & 0 \\
0 & 0 & 0 & 0 & 0 & m & 0 & 0 \\
0 & 0 & 0 & 0 & 0 & 0 & \bar{m} & 0 \\
0 & 0 & 0 & 0 & 0 & 0 & 0 & 0 \\
0 & 0 & 0 & 0 & 0 & 0 & 0 & 0 \\
0 & \bar{m} & 0 & 0 & 0 & 0 & 0 & 0 \\
0 & 0 & m & 0 & 0 & 0 & 0 & 0 \\
0 & 0 & 0 & 0 & 0 & 0 & 0 & 0
\end{array}\right) .
$$

The potential and the tensions of the fundamental string and of the D1-string are all given in terms of the vielbein $V_{A B}{ }^{a b}$, where $a, b$ are a couple of antisymmetric symplectic-traceless indices $a, b=1, \ldots, 8$, and representing the $\underline{27}$ of $U S p(8)$. This field, being an element of $E_{6} / U S p(8)$, carries both the indices $A, B=1, \ldots, 8$ of the $\underline{27}$ of $E_{6}$ and the indices $a, b=1, \ldots, 8$ of the $\underline{27}$ of $U S p(8)$. One can pass from the $E_{6}$ basis to the $U S p(8)$ one using the $S O(7)$ gamma matrices, $\Gamma$, defined in the Appendix of [15].

In our basis the vielbein is simply obtained by multiplying on the right the matrix $U$ by the following vector of gamma matrices:

$$
\left(\frac{\gamma_{i \bar{\jmath}}}{4 \sqrt{2}}, \frac{\epsilon_{i j k} \gamma_{\bar{\jmath} \bar{k}}}{4 \sqrt{2}}, \frac{\epsilon_{\bar{\imath} \bar{\jmath} \bar{k}} \gamma_{j k}}{4 \sqrt{2}}, \frac{\gamma_{i \bar{\jmath}}}{4 \sqrt{2}}, \frac{\gamma_{i}\left(1-\Gamma_{0}\right)}{4}, \frac{\gamma_{\bar{\imath}}\left(1-\Gamma_{0}\right)}{4}, \frac{\gamma_{i}\left(1+\Gamma_{0}\right)}{4}, \frac{\gamma_{\bar{\imath}}\left(1+\Gamma_{0}\right)}{4}\right) .
$$

Here the complex indices $i, \bar{\imath}$ run from 1 to 3 , and the new complex gamma matrices, $\gamma_{i}$ and $\gamma_{\bar{\imath}}$, are related to the real matrices of [15] by

$$
\begin{aligned}
\gamma_{1} & =\frac{\Gamma_{1}+i \Gamma_{2}}{\sqrt{2}}, \ldots \\
\gamma_{\overline{1}} & =\frac{\Gamma_{1}-i \Gamma_{2}}{\sqrt{2}}, \ldots
\end{aligned}
$$

The computation of the potential is now only a matter of straightforward gamma matrix algebra: just plug $V_{A B}^{a b}$ in the expression for the potential [15]

$$
V=-\frac{1}{32} g^{2}\left[2 W_{a b} W^{a b}-W_{a b c d} W^{a b c d}\right]
$$

where

$$
W_{a b c d}=\epsilon^{\alpha \beta} \eta^{I J} V_{I \alpha a b} V_{J \beta a b} .
$$


In these equations the $U S p(8)$ indices are raised and lowered with the matrix $\Gamma_{0}$, as shown in ref. [15.

Two features of the potential are worth mentioning. First, the potential does not depend on the dilaton, since the factors involving $\exp X_{0}$ cancel out. Moreover, the potential is quartic in the matrix $U$; therefore, one expects an asymptotic dependence on the field $m$ of the form

$$
V \sim e^{4 m}
$$

Similarly, the kinetic terms for the 12 antisymmetric tensors $B_{\mu \nu}^{I \alpha}$ are 15

$$
-\frac{1}{8} B_{\mu \nu a b} B^{\mu \nu a b}+\frac{1}{8 g} \epsilon^{\mu \nu \rho \sigma \tau} \eta_{I J} \epsilon_{\alpha \beta} B_{\mu \nu}^{I \alpha} D_{\rho} B_{\sigma \tau}^{J \beta}
$$

with $B_{\mu \nu}^{a b}=B_{\mu \nu}^{I \alpha} V_{I \alpha}^{a b}$.

Consider here the simpler case where only the dilaton is turned on. This has some interest in itself since for the theory at the $\mathrm{N}=8$ supersymmetric vacuum all scalars but the dilaton are zero. The matrix $U$ reduces to

$$
U=e^{X_{0}}
$$

with $X_{0}$ given in Eq. (B.8), and we obtain for $V_{A B}{ }^{a b}$

$$
\begin{aligned}
V_{I+}{ }^{a b} & =\frac{1}{4}\left(\cosh \rho+\sinh \rho e^{i \alpha}\right) \Gamma_{I}-\frac{1}{4}\left(\cosh \rho-\sinh \rho e^{i \alpha}\right) \Gamma_{I} \Gamma_{0}, \\
V_{I-}{ }^{a b} & =\frac{1}{4}\left(\cosh \rho+\sinh \rho e^{-i \alpha}\right) \Gamma_{I}+\frac{1}{4}\left(\cosh \rho-\sinh \rho e^{-i \alpha}\right) \Gamma_{I} \Gamma_{0} .
\end{aligned}
$$

In the real basis for $S L(2 ; R)$ we find, considering for simplicity a zero axion field $\alpha$,

$$
\begin{aligned}
V_{I 1}{ }^{a b} & =\frac{1}{2 \sqrt{2}}(\cosh \rho+\sinh \rho) \Gamma_{I}, \\
V_{I 2}{ }^{a b} & =\frac{i}{2 \sqrt{2}}(\cosh \rho-\sinh \rho) \Gamma_{I} \Gamma_{0} .
\end{aligned}
$$

We see that the matrix $A_{I \alpha, J \beta}=V_{I \alpha}{ }^{a b} V_{J \beta a b}$, which appears in the Lagrangian in the quadratic terms for $B_{\mu \nu}^{I \alpha}$, is diagonal both in the $S O(6)$ and the $S L(2 ; R)$ indices. The square of the tensions can be read from the restricted matrices $A_{I 1, J 1}$ and $A_{I 2, J 2}$. Not surprising, the eigenvalues are $e^{2 \rho}, e^{-2 \rho}$.

It is quite tedious, but straightforward to compute the matrix $A$ for non-zero $m$. The result is (for zero axion field),

$$
A_{I \alpha, J \beta}=\frac{\cosh (2 m)+1}{2} \Delta_{\alpha \beta} \delta_{I J}+\frac{\cosh (2 m)-1}{2} \epsilon_{\alpha \beta} J_{I J},
$$

where $J_{I J}=\operatorname{diag}\left(i \sigma_{2}, i \sigma_{2}, i \sigma_{2}\right)$ is a $6 \times 6$ matrix, and $\Delta=\operatorname{diag}[\exp (2 \rho)$, $\exp (-2 \rho)]$. The offdiagonal terms are irrelevant for our computation, since they modify the mass matrix of the 
antisymmetric tensors, but not their kinetic term. The result for the tensions, is $e^{m+\rho}, e^{m-\rho}$. We see that the contribution of the scalar is the same for both type of tensions, and, due to the fact that $A$ is quadratic in $U$, has the asymptotic form $e^{m}$. This result applies to other scalars as well.

\section{References}

[1] G. 't Hooft, Nucl. Phys. B72 (1974) 461

[2] J. Maldacena, Adv. Theor. Math. Phys. 2 (1998) 231, hep-th/9711200.

[3] S.S. Gubser, I.R. Klebanov and A.M. Polyakov, Phys.Lett. B428 (1998) 105, hepth/9802109.

[4] E. Witten, Adv. Theor. Math. Phys. 2 (1998) 253, hep-th/9802150.

[5] N. Itzhaki, J. Maldacena, J. Sonnenschein and S. Yankielowicz, Phys. Rev. D58 (1998) 046004, hep-th/9802042.

[6] E.Witten, Adv. Theor. Math. Phys. 2 (1998) 505, hep-th/9803131.

[7] J. Russo, hep-th/9808117; C. Csaki, Y. Oz and J.Russo, Phys. Rev. D59 (1999) 065008, hep-th/9810186.

[8] A. Kehagias and K. Sfetsos, hep-th/9902125; S. Gubser, hep-th/9902155.

[9] I.R. Klebanov and A.A. Tseytlin, hep-th/9811035; hep-th/9812089; hepth/9901101.

[10] J.A. Minahan, hep-th/9902074.

[11] A. Polyakov, Nucl. Phys. Proc. Suppl. 68 (1998) 1, hep-th/9711002.

[12] A. Polyakov, hep-th/9809057.

[13] H. J. Kim, L. J. Romans and P. van Nieuwenhuizen, Nucl. Phys. B242 (1984) 377.

[14] M. Günaydin, L.J. Romans and N.P. Warner, Phys. Lett. B154 (1985) 268; M.

Pernici, K. Pilch and P. van Nieuwenhuizen, Nucl. Phys. B259 (1985) 460.

[15] M. Günaydin, L.J. Romans and N.P. Warner, Nucl. Phys. B272 (1986) 598.

[16] P. Breitenlohner and D.Z. Freedman, Ann. Phys. 144 (1982) 249. 
[17] L. Girardello, M. Petrini, M. Porrati and A. Zaffaroni, JHEP 12 (1998) 022, hepth/9810126.

[18] J.Distler and F.Zamora, hep-th/9810206.

[19] A. Khavaev, K. Pilch and N.P. Warner, hep-th/9812035.

[20] E. D'Hoker and D.Z. Freedman, hep-th/9811257.

[21] B. de Wit and H. Nicolai, Nucl. Phys. B281 (1987) 211; B. de Wit, H. Nicolai and N.P. Warner, Nucl. Phys. B255 (1984) 29.

[22] S.W. Hawking and N. Turok, Phys. Lett. B425 (1998) 25, hep-th/9802030.

[23] J. M. Maldacena, Phys. Rev. Lett. 80 (1998) 4859, hep-th/9803002.

[24] J.A. Minahan, hep-th/9811156.

[25] L.D. Landau and E.M. Lifshitz, Quantum Mechanics (Pergamon Press, Oxford, III edition, 1976).

[26] S. Ferrara, M.A. Lledó and A. Zaffaroni, Phys. Rev. D58 (1998) 105029, hepth/9805082.

[27] S. J. Rey and J. Yee, hep-th/9803001; S. J. Rey, S. Theisen and J. Yee, Nucl. Phys. B527 (1998) 171, hep-th/9803135.

[28] A. Brandhuber, N. Itzhaki, J. Sonnenschein and S. Yankielowicz, Phys.Lett. B434 (1998) 36, hep-th/9803137; JHEP 9806 (1998) 001, hep-th/9803263. 